\title{
Frecuencia y patrón cambiante del cáncer pulmonar en México
}

Frumencio Medina-Morales, M.C., ${ }^{(1)}$ Margarita Salazar-Flores, M.C. ${ }^{(2)}$

Medina-Morales F, Salazar-Flores M. Frecuencia y patrón cambiante del cáncer pulmonar en México. Salud Publica Mex 2000;42:333-336.

\begin{abstract}
Resumen
Objetivo. Conocer la frecuencia, el tipo histológico y los cambios en cáncer pulmonar (CP) observados durante 40 años, en el Instituto $\mathrm{N}$ acional de Enfermedades Respiratorias (IN ER). Material y métodos. Estudio transversal realizado en el año de 1999. Se revisaron los informes de labores del IN ER, de 1983 a 1996, y los archivos de Patología Q uirúrgica, de 1957 a 1996. Se obtuvieron los datos de 1961 pacientes con los que se calcularon medidas de frecuencia acerca de: el lugar que ocupa el CP en los ingresos al Instituto, los estudios histológicos, edad, sexo, espécimen y diagnóstico. Resultados. Entre 1983 y 1996, el CP ocupó del octavo al segundo lugar en los ingresos al INER. LOS resultados se dividieron por décadas, en las dos primeras (1957-1976), 9\% fueron mujeres; en la tercera (1977-1986), $32 \%$, y en la última (1986-1996), 38\%. El carcino ma epidermoide predominó en las dos primeras décadas (61\%) y el adenocarcinoma en las dos últimas con 41 y $62 \%$, respectivamente. Conclusiones A ctualmente el CP es más frecuente en mujeres y predomina el adenocarcinoma.
\end{abstract}

Palabras clave: neoplasmas pulmonares/anatomía \& histología; México
Medina-Morales F, Salazar-Flores M.

The frequency and changing pattern

of lung cancer in Mexico.

Salud Publica Mex 2000;42:333-336.

\begin{abstract}
A bstract
Objective. To assess the frequency, histological type and the epidemio logical pattern of lung cancer (LC), during a 40 year period, at the $N$ ational Institute of Respiratory $D$ iseases (IN ER). Material and methods. This cross-sectional study was conducted in 1999. Data were abstracted from the IN ER's service reports for 1983-1996 and the surgical pathology archives for 1957-1996. D ata from 1961 patients were analyzed, to obtain frequency measures on: the characteristics of IN ER LC patient admissions, age, sex, histological studies, specimens and diagnoses. Results. Between 1983-1996, LC ranked eighth to second place in IN ER inpatient admissions. Results were grouped by decades. In the first two decades (1957-1976), 9\% of patients were female; $32 \%$ in the third (1977-1986), and 38\% in the last decade (1987-1996). Epidermoid cell carcinoma was more frequent in the two first decades $(61 \%)$ and adenocarcinoma in the last two, (41\% and $62 \%$, respectively.) Conclusions $\mathrm{LC}$ is currently more frequent in women, and adenocarcinoma is the most frequent neoplasia.
\end{abstract}

Key words: lung neoplasms/anatomy \& histology; Mexico
$\mathrm{E}$ 1 cáncer pulmonar en la actualidad es el tumor maligno más frecuente en el mundo. En los Estados Unidos de América (EUA), en 1996, fue la principal causa de muerte en ambos sexos. Antes del siglo XX, el cáncer pulmonar era una entidad patológica muy rara. A partir de 1930, su frecuencia ha aumentado. Se estima que para el año 2025 se incrementará el número de muertes en más de $80 \%$, es decir, a tres y medio millones en países en desarrollo. ${ }^{1-5}$

A partir de 1970, ha habido un incremento en la frecuencia de muertes por cáncer pulmonar en las mujeres y en pacientes jóvenes y un cambio en el tipo

(1) Area clínica, Instituto Nacional de Enfermedades Respiratorias (IN ER). Facultad de Medicina, Universidad N acional Autónoma de México, México.

(2) Subdirección de Educación Continua, Dirección de Enseñanza, IN ER, México.

Fecha de recibido: 13 de septiembre de 1999 - Fecha de aprobado: 8 de mayo de 2000

Solicitud de sobretiros: Dr. Frumencio Medina Morales. Instituto N acional de Enfermedades Respiratorias. Calzada de Tlalpan 4502, sección XVI, 14080 México, D.F., México. 
histológico; actualmente el adenocarcinoma es más común que el carcinoma epidermoide..$^{6-9}$

Asimismo, la asociación entre tabaco y cáncer de pulmón ha sido bien establecida, el riesgo relativo en fumadores se ha duplicado en hombres y cuadruplicado en mujeres. Existe una predisposición genética, los fumadores con antecedentes familiares de cáncer pulmonar tienen un riesgo relativo de 2 a 2.5 veces mayor en relación con fumadores sin antecedentes familiares. La dieta es otro factor, el riesgo se incrementa con una dieta alta en colesterol y en consumo de grasas; se ha mencionado un efecto protector de las vitaminas A y C y los betacarotenos. ${ }^{9-12}$

En México es difícil evaluar la frecuencia del padecimiento. Datos de la Secretaría de Salud (SSA) indican que la mortalidad por cáncer se incrementó de $1.78 \%$, en 1950 , a $9.32 \%$ (tasa bruta por 10000 habitantes), en $1986 .{ }^{13}$ En 14824 autopsias practicadas, entre 1953 y 1970, en los principales hospitales generales de la ciudad de México, de la entonces Secretaría de Salubridad y Asistencia (SSA), del Instituto Mexicano del Seguro Social (IMSS) y del Instituto de Seguridad y Servicios Sociales de los Trabajadores del Estado (ISSSTE), y en el Hospital General de México-SSA, las neoplasias malignas fueron la enfermedad principal en $28.5 \%$ y, de éstas, el cáncer pulmonar ocupó el tercer lugar con $7.4 \%$. En el IMSS las enfermedades se dividieron por aparatos y sistemas, el aparato respiratorio ocupó el sexto sitio y las neoplasias malignas de éste representaron 9\%; en el ISSSTE, los tumores malignos ocuparon el segundo lugar como enfermedad principal con $17 \%$ y, de éstos, el cáncer broncogénico representó $14 \%{ }^{14}$

De 1981 a 1985, el carcinoma pulmonar ingresó al grupo de las primeras 20 causas de muerte, y aunque la tasa de mortalidad general disminuyó la de cáncer pulmonar mostró una tendencia al aumento. ${ }^{15}$ En 1976, en el IMSS, entre las defunciones por tumores malignos, el carcinoma cervicouterino ocupaba el primer lugar; en 1982, el cáncer pulmonar lo desplazó y alcanzó el primer puesto. ${ }^{16}$

La tasa cruda de mortalidad por cáncer pulmonar (por 100000 habitantes) se incrementó de 5.01, en 1979, a 7.25, en 1993, y esto fue más ostensible en los estados del norte de la República mexicana. ${ }^{17}$

Alcántara y colaboradores ${ }^{18}$ analizaron 1211 protocolos de autopsias del Hospital General de México, de sujetos mayores de 60 años, estudiados en dos periodos (1960-1965 y 1981-1985), y encontraron disminución de algunas enfermedades como la amibiasis. Las neoplasias malignas persistieron en ambos periodos, el cáncer pulmonar que ocupaba el segundo lugar en los años sesenta, pasó en los ochenta al primer sitio.
Por su parte, Ramírez y colaboradores, del Hospital General de México, ${ }^{19}$ informaron de 923 casos de carcinoma broncogénico, estudiados entre 1971 y 1990; la relación hombre/mujer fue de 1.95:1. El tipo histológico fue epidermoide en $32 \%$; adenocarcinoma, en $28 \%$, y carcinoma de células pequeñas, $13 \%$ en hombres; en mujeres, adenocarcinoma, en 39\% y epidermoide, en $29 \%$. Asimismo, Medina y colaboradores, ${ }^{20}$ en el Instituto Nacional de Enfermedades Respiratorias (INER), encontraron una alta frecuencia en pacientes de entre 61 y 70 años, el tipo histológico más frecuente fue el adenocarcinoma.

El objetivo del presente trabajo fue conocer la frecuencia de los cambios en el tipo histológico de cáncer pulmonar en el lapso de 1957 a 1996.

\section{Material y métodos}

Estudio transversal realizado en el año de 1999. Se revisaron los informes de labores del INER, de 19831996, y los archivos de Patología Quirúrgica, de 1957-1996. De los informes anuales de labores se obtuvieron los siguientes datos: el lugar que ocupa el cáncer pulmonar en los ingresos al propio Instituto, el porcentaje de muertes por cáncer pulmonar y el lugar que ocupa en relación con otras enfermedades. De los archivos de Patología Quirúrgica se obtuvieron los siguientes datos: número de registro de patología, nombre, edad, sexo, espécimen y tipo histológicos.

Se analizaron los datos de 1961 pacientes atendidos en el INER en el lapso de 1957 a 1996.

\section{Resultados}

El cáncer pulmonar ocupó del octavo al segundo lugar entre los pacientes que ingresaron al INER, entre 1983 y 1996, y representó de 3.9 a 10.3\%; en cuanto al número de muertes por dicha enfermedad, con relación a otras enfermedades, y durante el mismo lapso, ocupó entre el cuarto y el primer lugar y el porcentaje varió de 8.6 a 19.1 (cuadro I).

En 40 años (1957-1996) se estudiaron 1961 pacientes, los resultados fueron divididos por décadas, de 1957 a 1966 se estudiaron 33 pacientes; de 1967 a 1976, 102; de 1977 a 1986, 499, y de 1987 a 1996, 1327.

En cuanto al tipo histológico el carcinoma epidermoide varió su frecuencia como sigue: $61,61,36$ y $26 \%$, respectivamente, en las cuatro décadas, y el adenocarcinoma varió en los mismos periodos de 21,14, 41 y $62 \%$ (figura 1 ).

En cuanto al sexo, de los 1961 casos, 36\% fueron mujeres. Cuando analizamos estos datos por décadas 
Cuadro I

TUMORES MALIGNOS DEL A PARATO RESPIRATORIO en el Instituto N acional de Enfermedades Respiratorias. México, 1983-1996

\begin{tabular}{llccc} 
Año & Morbilidad \% & Lugar TM & Mortalidad\% & Lugar TM \\
1983 & 3.9 & 70. & 8.6 & $2 \mathrm{do}$. \\
\hline 1984 & 5.9 & 70. & 13.5 & $2 \mathrm{do}$. \\
\hline 1985 & 3.79 & 80. & 8.56 & 30. \\
\hline 1986 & 7.3 & 50. & 14.1 & $2 \mathrm{do}$. \\
\hline 1987 & 5.9 & 50. & 13.45 & $2 \mathrm{do}$. \\
\hline 1988 & 6.5 & 40. & 10.4 & 30. \\
\hline 1989 & 8.5 & 30. & 16.7 & 20. \\
\hline 1990 & 7.3 & 50. & 16.5 & 20. \\
\hline 1991 & 6.4 & 60. & 10.8 & 40. \\
\hline 1992 & 6.7 & 50. & 11.19 & 40. \\
\hline 1993 & 8.6 & 30. & 14.4 & 10. \\
\hline 1994 & 9.8 & 30. & 16.2 & 10. \\
\hline 1995 & 9.8 & 20. & 19.1 & 10. \\
\hline 1996 & 10.3 & 30. & 13.2 & 30.
\end{tabular}

Referencia 21

*TM = tumores malignos

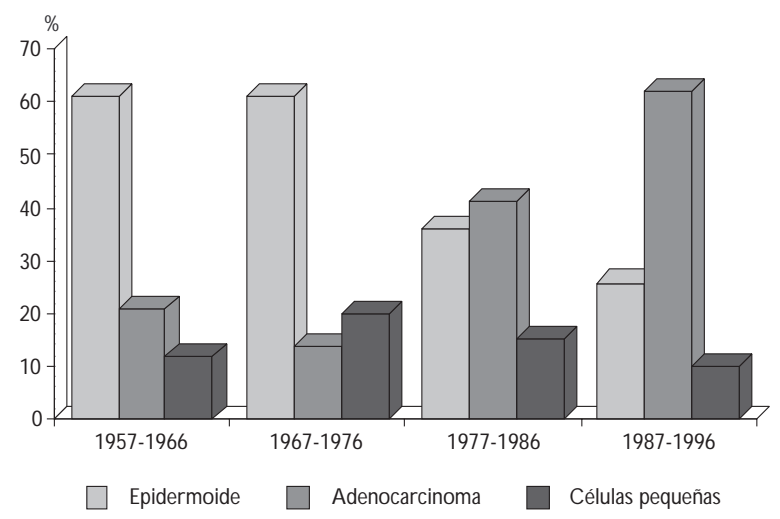

Figura 1. Evolución del CánCer pulmonar. Instituto Nacional de Enfermedades Respiratorias, México, 1957-1996

encontramos lo siguiente: entre 1957 y 1966, 9\% fueron mujeres; de 1967 a 1976, 28\%; de 1977 a 1986, 32\%, y en la última década (1987-1996), 38\%.

El $72 \%$ de los pacientes con cáncer pulmonar tenían edades comprendidas entre 51 y 80 años. Ese mismo porcentaje de aquéllos eran fumadores y $28 \%$, no fumadores.

\section{Discusión}

En las variaciones en frecuencia debemos tomar en consideración que durante las dos primeras décadas (1957-1976) y parte de la tercera (1977-1986) el Hospital atendía principalmente a pacientes con tuberculosis y, por lo tanto, los casos de cáncer pulmonar eran muy escasos, sólo en la última década, ya como Instituto Nacional de Enfermedades Respiratorias, se atendió a pacientes con problemas respiratorios en general.

El aumento en la frecuencia del cáncer pulmonar encontrado en esta serie, lo podemos comparar con lo publicado previamente en la literatura especializada. ${ }^{1-11,13,15-21}$

Vincent y colaboradores, ${ }^{8}$ en un estudio que duró 13 años (1962-1975), informan que en 1682 pacientes hubo cambios en la frecuencia del carcinoma epidermoide, pues descendió de 48.6 a $25.5 \%$ en ese periodo, así como del adenocarcinoma, mismo que se incrementó de $17.6 \%$, en 1962 , a $29.8 \%$, en 1975 ; paralelamente, hubo un cambio respecto al sexo, en 1962, $90.5 \%$ de los pacientes fueron hombres, y $9.5 \%$, mujeres; en 1975, las mujeres representaron $21.3 \%$.

En el material, semejante a otras series publicadas, ${ }^{8,20}$ hubo un aumento en la frecuencia del cáncer pulmonar en las mujeres, mientras en las décadas de 1957-1976 sólo representaron 9\%, en la última década (1987-1996) alcanzaron 38\%.

El-Torky y colaboradores, ${ }^{21}$ en 1990, publicaron sus resultados de un estudio de 4928 pacientes estudiados en un periodo de 22 años (1964-1985). Hicieron notar el aumento en la frecuencia del cáncer pulmonar con relación a otras neoplasias; en 1964, el cáncer de pulmón representaba 11.9\% y, en 1985, 17.1\%. En 1964, el carcinoma epidermoide representaba $44 \%$; el adenocarcinoma, $13 \%$ y, en 1984, el carcinoma epidermoide bajó a 32\% y el adenocarcinoma aumentó a $31 \%$. En 1951, la tasa de mortalidad por cáncer fue de 25 por 100000 habitantes, mientras que en 1973 aumentó a 60 .

En países donde el tabaquismo es un hábito éste es responsable de 80 o $90 \%$ de las muertes por esta enfermedad. ${ }^{7}$ Por los años treinta se observó que el cáncer pulmonar era más frecuente en las ciudades que en el campo y se consideró que podría atribuirse a la contaminación atmosférica; investigaciones posteriores han demostrado que la contaminación sólo es responsable de $10 \%$ de los casos y que $90 \%$ pueden atribuirse al tabaquismo. ${ }^{1}$

El tabaquismo está aumentando rápidamente en los países en desarrollo, por lo que ocho de cada 10 con el más alto consumo de tabaco son países en desa- 
rrollo, y de los 30 con más alto consumo sólo nueve son industrializados. ${ }^{1}$

Encontramos el antecedente de hábito tabáquico en $72 \%$ de los casos, es muy difícil evaluar el tabaquismo pasivo. Alonso y colaboradores 22 encontraron el antecedente de tabaquismo en $65 \%$ de 612 casos de cáncer pulmonar estudiados por autopsia, entre 1953 y 1996.

A pesar de que se pueden prevenir más de $80 \mathrm{o}$ $90 \%$ de los cánceres y menos de $15 \%$ son curables, actualmente la mayor parte de los recursos y actividades profesionales están enfocados hacia el tratamiento y no hacia la prevención. ${ }^{1}$

\section{Referencias}

1. Stanley K, Stjernsward J. Lung cancer. A worldwide health problem. Chest 1989;96:1S-5S.

2. Frank AL. Epidemiology and etiology of lung cancer. Clin Chest Med 1982:3:2119-2122.

3. C arter D, Eggleston JC. Tumors of the lower respiratory tract. A tlas of tumor pathology. W ashington, D.C.: Armed Forces Institute of Pathology, 1980;(Second series. Fasc. 17).

4.A skin FB. Lung neo plasm. Posgraduate Course 15. Pulmonary pathology for the pulmonologist. San Franscisco (CA):A merican Thoracic Society (ATS), 1997.

5. Mohar BA, Frías MM. Epidemiología del cáncer broncogénico en México. N eumol Cir Tor 1993;52:19-30.

6. Colby TV, Koss MN , Travis W D. Tumors of the lower respiratory tract. Atlas of tumor pathology. W ashington, D.C.: Armed Forces Institute of Pathology, 1995;(Third series. Fasc 13).

7. Parkin DM. Trends in lung cancer incidence worldwide. Chest 1989; 96:5S-7S.
8.Vincent RG, Pickren JW, Lane W W, Bross I,Takita H, H outen L et al.The changing histopathology of lung cancer. A review of 1682 cases. Cancer 1977;39:1647-1655.

9. D avila D G, W illiams DE. Symposium on intrathoracic neoplasms (part I). The etiology of lung cancer. Mayo Clin Proc 1993;68:170-182.

10. Ibarra-Pérez C, C riales-C ortés JL, Cicero SR, G arcía-Sainz M. A vances recientes en carcinoma broncogénico. Gac Med Mex 1991;127:47-63.

11. BeckettW S. Epidemiology and etiology of lung cancer. C lin C hest Med 1993;14:1-15.

12. Miller AB, Harve AR. D iet and lung cancer, vitamin A and B carotenos. Chest 1989;96:8S-9S.

13. Benítez LB, Q uiñones SG . El cáncer. ¿Un problema de salud en México? Rev Med Inst Mex Seguro Soc 1991;29:195-214.

14. Márquez MH,A mbrosius DK, Flores-Barroeta F,Velazco-Avilés F, Salas MM. Principales padecimientos encontrados en las necropsias de algunos hospitales de la ciudad de México. Gac Med Mex 1971;102:191-215.

15. Rivero-Serrano 0, Fortul Van Der Goes T, G reen IS. Algunas reflexiones sobre el diagnóstico y la prevención del cáncer broncogénico. Gac Med Mex 1991;127:41-46.

16. Casab HG, Barroso E, Santiago PH, Rendón J. Progresos en el registro del cáncer en el Instituto Mexicano del Seguro Social. Rev Med Inst Mex Seguro Soc 1986;24:125-131.

17. Lazcano-Ponce CE,Tovar GV, Meneses GF, Rascón-Pacheco RA, Hernández-Avila M. Trends of lung cancer mortality in Mexico. Arch Med Res 1997;28:565-570.

18.Alcántara-Vázquez A, A mancio-C hassin 0 , G onzález-C hávezA, Hernández-González M, Carrillo-Villa H. Mortalidad en pacientes geriátricos del Hospital General de México. Salud Publica Mex 1993;35:368-375.

19. Ramírez E, Cicero R, Zúñiga $G, N$ ovelo $V, N$ avarro $F$, C asanova JM. Bronchogenic cancer at the Hospital General de Mexico.A study of two decades. Salud Publica Mex 1995; 37:155-161.

20. Medina F, Barrera R, Morales J, Echegoyen R, Chavarría J, Rébora F. Primary lung cancer in Mexico city:A report of 1019 cases. Lung $C$ ancer 1996;14:185-193

21. El-Torky M, El-Zeky F, Hall JC. Significant changes in the distribution of histologic types of lung cancer. C ancer 1990;65:2361-2363.

22.Alonso-de Ruiz P, Rodríguez-Martínez HA, Ponciano G , Rivero-de Jesús V.Tendencia del cáncer pulmonar en el Hospital General de México. Estudios de autopsias de 1953 a 1996. Patologia (Mex) 1997;35:S15. 